\title{
Ransomware Detection Using Markov Chain Models Over File Headers
}

\author{
Nicolas Bailluet ${ }^{1}$, Hélène Le Bouder ${ }^{2}$ and David Lubicz ${ }^{3}$. \\ ${ }^{1}$ ENS Rennes, France \\ ${ }^{2}$ OCIF IMT Atlantique Campus Rennes, France \\ ${ }^{3}$ DGA MI, Bruz, France
}

Keywords: $\quad$ Ransomware, Detection, Malware, Markov Chain, File Header

Abstract: In this paper, a new approach for the detection of ransomware based on the runtime analysis of their behaviour is presented. The main idea is to get samples by using a mini-filter to intercept write requests, then decide if a sample corresponds to a benign or a malicious write request. To do so, in a learning phase, statistical models of structured file headers are built using Markov chains. Then in a detection phase, a maximum likelihood test is used to decide if a sample provided by a write request is normal or malicious. We introduce new statistical distances between two Markov chains, which are variants of the Kullback-Leibler divergence, which measure the efficiency of a maximum likelihood test to distinguish between two distributions given by Markov chains. This distance and extensive experiments are used to demonstrate the relevance of our method.

\section{Introduction}

Ransomware are a family of malware that ask a payment to the legitimate users for accessing their machine or computer files. They are one of the most serious security threats on the Internet. The number of attacks has increased drastically these last years, resulting in huge losses and disruptions. There are two types of ransomware: some prevent the usage of the computer, others encrypt files. In this paper we are interested in the last case called crypto-ransomware. Crypto-ransomware are more and more efficient and easy to operate by malicious individuals and organisations. They are provided as ready-to-use toolkit with robust cryptographic algorithms, embed evasion techniques to defeat intrusion detection and are capable to spread in a network to infect many computers.

In order to mitigate this threat, it is important to be able to detect and stop automatically the malicious activity of ransomware.

In this paper, we present a new method to detect ransomware that belongs to the class of behavioural approach which consists in detecting malign activity : the encryption data, by distinguishing it from normal activity.

In this paper, we explain:

- how to use Markov chains in order to build a statistical model of a normal behaviour by training it with write requests;

- how to compute maximum likelihood tests that distinguish samples drew following two distributions well described by Markov chains;

- we report on extensive experiments to demonstrate the relevance of our method.

The main originality of the paper, which is developed in Section 4, is related to the remark that the Kullback-Leibler differential entropy measures the efficiency of the maximum likelihood test to distinguished the distributions given by two Markov chains. Unfortunately, the Kullback-Leibler entropy can not always be computed in our case because the probability of some transition is 0 . So we have to tweak a little bit the definition of Kullback-Leibler entropy to obtain a useful criterion. This criterion is then used as an essential tool to conduct our experiment methodology and adjust the parameters of the Markov chain so as to maximise the probability of detection.

The state of the art of countermeasures are described in section 2 In section 3 , the basic definition and main theoretical ingredients that are used in the paper, are introduced. Section 4 is devoted to a preliminary analysis of the data. In section 5, we present the results of our first experiments to then refine our approach in Section 6. The Section 7 tests our approach against real-world ransomware-encrypted files. Finally, conclusions are drawn in section 8 


\section{State of the art}

Different surveys have been published on ransomware protections (Genç et al., 2018; Al-rimy et al., 2018b; Aurangzeb et al., 2017, Moussaileb, 2020). A first idea to protect data against ransomware attacks is to use data back-up as explained in (Castiglione and Pavlovic, 2019: Baykara and Sekin, 2018) but it is not possible to have at any time an up to date back-up of data and to decide when and which data need to be restored it is necessary to have a mechanism to detect a ransomware attack when it occurs.

The classic detection techniques used for all malware, such as as NIDS (Network Intrusion Detection Systems), can be used for ransomware. Signature based detection remains a valid way to detect an infection. Network traffic is compared to previously well-known malware patterns, called signatures as in (Moussaileb et al., 2019, Ahmadian et al., 2015. Cabaj et al., 2018; Almashhadani et al., 2019). The limitation of these techniques is that they are unable to detect new malware, never seen before. This is a serious problem with ransomware because a lot of attacks come from new ransomware.

The detection of specific behaviours of ransomware are used too. Some detection mechanisms specific to ransomware can be to watch how files are touched (Moussaileb et al., 2018) or to detect ransom note writing (Yassine Lemmou, 2019). Some papers studies the transaction for the ransom payment (Akcora et al., 2019). But, the most specific behaviour of ransomware is to overwrite files with encrypted data so that these methods still generate a lot of false alarms.

Often ransomware do not come with their own cryptographic libraries and rather rely on the victims own tools. So many protections are based on controlling accesses over the cryptographic tools in Windows (Palisse et al., 2016; Chen et al., 2017; Al-rimy et al., 2018a; Al-rimy et al., 2019). One other way to detect ransomware is to have honey pots. These are files that trigger an alarm when they are modified (Moore, 2016; Patton et al., 2019).

Finally there is a family of ransomware detection mechanisms which rely on encryption detection. Indeed encryption produces data which have a pseudorandom distribution, therefore increasing the entropy of the files. There is an important literature about statistical tests of randomness which can be used to detect encrypted data (Scaife et al., 2016, Continella et al., 2016, Kharraz and Kirda, Palisse et al., 2017, Kharraz et al., 2016).

A recent study (Pont et al., 2020) explains in detail why the classical statistic tool are not sufficient to detect ransomware. That is why in this paper a new approach is presented.

A drawback of these methods is that randomness behaviour is not a complete characterisation of encrypted streams: compressed data streams which are quite common behave in the same manner. As a consequence, in order to avoid too much false alarms it is necessary to combine detection mechanism based on randomness with other criteria, specific of ransomware malign activity.

Another problem on the statistical level is that we want to distinguish malign activity from normal behaviour. But if we have a clear statistical model for malign activity, namely random data, we do not have its counterpart for normal behaviour. In this situation, the use of classical $\chi^{2}$ tests (Palisse et al., 2017) is awkward to say the least since they are meant to detect improbable events under the hypothesis that the stream of data is random where we actually want to single out improbable events in the case that the stream of data is a normal structured file. This makes it impossible to carry out a precise analysis of the efficiency of the statistical tests that we use for the detection of ransomware and to design the most efficient statistical tests.

In this paper, we present a new method to detect ransomware that belongs to the class of behavioural approach which consists in detecting malign activity by distinguishing it from normal activity. A well suited probe for the kind of malign activity involved by ransomware is provided by write requests which are handled by the kernel and is a mandatory call in order to perform a write access on a hard drive. These requests can be monitored by a specific driver running in kernel land, the operation of which can not be disrupted by the ransomware (which is running in user land).

Whereas most statistical models and machine learning techniques recently implemented focus on neural networks or other modern machine learning methods (Lee et al., 2019), we chose to use Markov chains to model the stream of data. Unlike modern machine learning methods, Markov chains are fairly easy to understand and manipulate. They induce well understood probability distributions and can be used in conjunction with a maximum likelihood test that is known to be the most powerful in a large class of statistical tests. In building the model, we mainly focus on file headers because they produce data stream of very structured data with recurrent patterns which make them very different from a statistical point of view from encrypted data. 


\section{Basic definitions and notations for Markov chains}

Let bit $=\{0,1\}$ be an alphabet of cardinal $2 . \forall k \in \mathbb{N}$, let $S_{k}$ be the set of finite sequences of length $k$ with coefficients in bit. The data stream is represented by a probability distribution on $S_{k}$. This probability distribution can be:

- either the uniform $U_{k}$ distribution which represents encrypted data;

- or an unknown distribution $D_{k}$ which represents normal header data.

We suppose that we can obtain samples drawn following the distribution $D_{k}$ (and of course also following the distribution $U_{k}$ ). So, we can obtain information about this distribution. The problems that we want to solve efficiently are:

1. obtain a model of the distribution $D_{k}$ by learning it from samples drawn following $D_{k}$;

2. for $s$ a given sample, decide from which distribution $D_{k}$ or $U_{k}$ it comes from.

In order to learn the distribution $D_{k}$, we use the model provided by Markov chains. It is a fundamental and widely used model in information theory and is particularly well suited to statistically model a data stream. There exists more general definition in the huge literature on the subject (see (Ash, 1990) for instance), but we have chosen in this paper to slightly adapt it to our needs.

Definition 3.1. We keep the previous notations for bit and $S_{m}$. Let $\mathcal{P}\left(S_{m}\right)$ be a set of subsets of $S_{m}$. The map succ is defined as :

$$
\begin{aligned}
\operatorname{succ}: S_{m} & \rightarrow \mathcal{P}\left(S_{m}\right) \\
s=s_{1} \ldots s_{m} & \mapsto\left\{s_{2} \ldots s_{m} b \mid b \in \mathbf{b i t}\right\} .
\end{aligned}
$$

A binary Markov chain $X$ with memory $m$ is a sequence $\left(X_{i}\right)_{i \geq 0}$ of random variables with value in $S_{m}$, the set of states, and such that $\forall n \geq k$ :

1. $\mathbb{P}\left(X_{n}=x_{n} \mid X_{n-1}=x_{n-1}, \ldots, X_{n-k}=x_{n-k}\right)=$ $\mathbb{P}\left(X_{n}=x_{n} \mid X_{n-1}=x_{n-1}\right)$,

2. $\mathbb{P}\left(X_{n}=x_{n} \mid X_{n-1}=x_{n-1}\right)$ does not depend on $n$.

We suppose moreover that if $x_{n} \notin \operatorname{succ}\left(x_{n-1}\right)$ then $\mathbb{P}\left(X_{n}=x_{n} \mid x_{n-1}=x_{n-1}\right)=0$.

It is clear that succ maps any element $s \in$ $S_{m}$ to the set of its possible successors. If $s^{\prime}=s_{1}^{\prime} \ldots s_{m}^{\prime} \in \operatorname{succ}(s)$ then the transition from $s$ to $s^{\prime}$ is labelled by $s_{m}^{\prime} \in$ bit.

A binary Markov chain is classically defined by the following data:

- the memory $m$ which is an integer;
- the transition matrix $T=t_{x, y}$ such that, $\forall x, y \in S_{m}$ :

$$
t_{x, y}=\mathbb{P}\left(X_{n}=x \mid X_{n-1}=y\right) .
$$

- an initial state $X_{0}$ which is a random variable with value in $S_{m}$.

Definition 3.2. An element $X_{k_{0}}$ of the Markov chain $\left(X_{i}\right)_{i \geq 0}$ is stable if, $\forall k \geq k_{0}: X_{k+1}=X_{k}$.

A Markov chain with memory $m$ verifies the ergodicity and irreducibility condition (Ash, 1990) so that by (Ash, 1990) we know that any such Markov chain has a unique stable element. A stable element gives by definition a random variable on $S_{m}$ that we denote by $\widehat{X}(m)$. More generally, it also defines random variable $\widehat{X}(k)$ on $S_{k}, \forall k>m$, by the induction (1) Because of Definition 3.1, the preceding formula does not depend on $n$. One has to remark that $\widehat{X}(m+1)$ allows to recover $\widehat{X}(m)$ using (2). But, it means that $\widehat{X}(m+1)$ allows to recover the transition matrix $t_{x, y}$, for $x \in \operatorname{succ}(y)$ using Equation (1) with $k=m+1$. The distribution $\widehat{X}(m+1)$ can then be encoded by a function

$$
\begin{aligned}
f_{\widehat{X}}: S_{m+1} & \rightarrow[0,1] \\
b_{1} \ldots b_{m+1} & \mapsto \mathbb{P}\left(\widehat{X}(m+1)=b_{1} \ldots b_{m+1}\right) .
\end{aligned}
$$

This way of encoding the transition matrix takes $O\left(2^{m}\right)$ memory bits and is more efficient that storing all the $t_{x, y}, \forall x, y \in S_{m}$ (which takes $O\left(2^{2 m}\right)$ memory bits).

Finally, we denote by $X\left(m, f_{\widehat{X}}, X_{0}\right)$ the Markov chain with memory $m$, transition matrix encoded by $f_{\widehat{X}}$ and initial state $X_{0}$. From the knowledge of $f_{\widehat{X}}$, one can recover the stable state $\widehat{X}(m)$ from (2). Then $\mathbb{P}\left(\widehat{X}\left(k_{0}\right)=s\right)$ for $k_{0}>m$ is given for $s=b_{1} \ldots b_{k_{0}} \in$ $S_{k_{0}}$ by 4 In the preceding formula, the first equality is obtained by an inductive application of (1) for $k=$ $k_{0}, k_{0}-1, \ldots, m+1$ and the second equality comes from (3).

\section{Modeling data stream using Markov chains}

We have a corpus of files with different extensions and we want to model the statistical properties of their header with Markov chains in order to be able to distinguish them from encrypted files. A Markov chain of memory $m$ is used and trained, with the first $N$ bits of each file. In this section, we explain how to train the model, compute the test and assess the efficiency of the test. 


$$
\begin{gathered}
\mathbb{P}\left(\widehat{X}(k)=b_{1} \ldots b_{k}\right)=\mathbb{P}\left(X_{n}=b_{k-m+1} \ldots b_{k} \mid X_{n-1}=b_{k-m} \ldots b_{k-1}\right) \cdot \mathbb{P}\left(\widehat{X}(k-1)=b_{1} \ldots b_{k-1}\right) . \\
\mathbb{P}\left(\widehat{X}(m)=b_{1} \ldots b_{m}\right)=\mathbb{P}\left(\widehat{X}(m+1)=b_{1} \ldots b_{m} 1\right)+\mathbb{P}\left(\widehat{X}(m+1)=b_{1} \ldots b_{m} 0\right) . \\
\mathbb{P}\left(\widehat{X}\left(k_{0}\right)=s\right)=\mathbb{P}\left(\widehat{X}(m)=b_{1} \ldots b_{m}\right) \prod_{i=m+1}^{k_{0}} \mathbb{P}\left(X_{n}=b_{i-m+1} \ldots b_{i} \mid X_{n-1}=b_{i-m} \ldots b_{i-1}\right) \\
=\mathbb{P}\left(\widehat{X}(m)=b_{1} \ldots b_{m}\right) \prod_{i=m+1}^{k_{0}} \frac{\mathbb{P}\left(\widehat{X}(m+1)=b_{i-m} \ldots b_{i}\right)}{\mathbb{P}\left(\widehat{X}(m)=b_{i-m} \ldots b_{i-1}\right)}
\end{gathered}
$$

\subsection{Training the Model}

Let $H_{N}$ be the list of the first $N$-bit sequences of each file in the corpus. Let $X\left(m, f_{\widehat{X}}, X_{0}\right)$ be the Markov chain which represents the best statistical approximation of the sample $H_{N}$. To define this Markov chain $X\left(m, f_{\widehat{X}}, X_{0}\right)$, we have to compute $f_{\widehat{X}}(s), \forall s \in S_{m+1}$ and $\mathbb{P}\left(X_{0}=s\right), \forall s \in S_{m} . \quad \forall s \in S_{m+1}$, let $c_{H_{N}}(s)$ be the occurrence count of $s$ in every sequence of $H_{N}$. $\forall s \in S_{m}$, let $c_{H_{N, 0}}$ be the number of sequences in $H_{N}$ such as $s$ is the $m$ first bits. Denote by $\left|H_{N}\right|$ the number of the list $H_{N}$ i.e the number of files. Finally, let $\left|H_{N}\right|(k)=(N-k) \cdot\left|H_{N}\right|$ be the number of sequences of bits of length $k$ in $H_{N}$. We have:

$$
\begin{aligned}
& \forall s \in S_{m+1}, f_{\widehat{X}}(s)=\frac{c_{H_{N}}(s)}{\left|H_{N}\right|(m+1)}, \\
& \forall s \in S_{m}, \mathbb{P}\left(X_{0}=s\right)=\frac{{ }^{H_{N, 0}}(s)}{\left|H_{N}\right|} .
\end{aligned}
$$

On the other side, let $X^{r}\left(m, f_{\widehat{X}^{r}}, X_{0}^{r}\right)$ be the Markov chain with memory $m$ which represents the best statistical approximation of an encrypted file. As the statistical behaviour the encrypted file is indistinguishable from the one of a perfectly random sequence (Katz and Lindell, 2007). Thus:

$$
\begin{aligned}
& \forall m \in S_{m+1}, f_{\widehat{X}^{r}}(s)=\frac{1}{2^{m+1}}, \\
& \forall m \in S_{m}, \mathbb{P}\left(X_{0}^{r}=s\right)=\frac{1}{2^{m}} .
\end{aligned}
$$

From (5) and (6), we obtain Algorithm 1 to compute a Markov chain model of the data stream associated to file headers.

\subsection{Testing Samples against models}

For $i \in\{1,2\}$, let $X^{i}\left(m, f_{\widehat{X}^{i}}, X_{0}^{i}\right)$ be two Markov chains. Let $s \in S_{N}$ be a sample header. We want to decide which of the two trained models $X^{i}\left(m, f_{\widehat{X}^{i}}, X_{0}^{i}\right)$ for $i=1,2$ is the more likely to match $s$. For this we apply the likelihood ratio test.

Definition 4.1. Likelihood Ratio Test for Trained Models. Let $s \in S_{k}$ be a sample, we define the likelihood ratio of $s$ with respect to $X^{i}\left(m, f_{\widehat{X}^{i}}, X_{0}^{i}\right)$ for

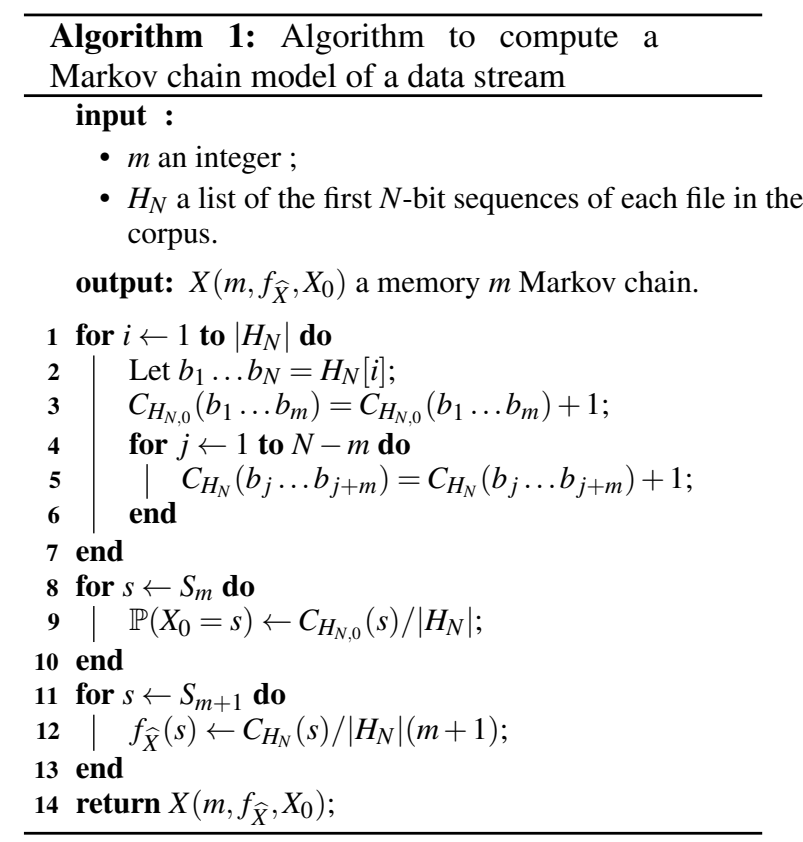

$i=1,2$, two Markov chains, as:

$$
\Lambda\left(s, \widehat{X}^{1}(k), \widehat{X}^{2}(k)\right)=\frac{\mathbb{P}\left(\widehat{X}^{1}(k)=s\right)}{\mathbb{P}\left(\widehat{X}^{2}(k)=s\right)} .
$$

The likelihood ratio test then is defined by:

$T\left(c, s, \widehat{X}^{1}(k), \widehat{X}^{2}(k)\right)=\left\{\begin{array}{ll}1 & \text { if } \Lambda\left(s, \widehat{X}^{1}(k), \widehat{X}^{2}(k)\right)>c \\ 0 & \text { otherwise }\end{array}\right.$.

Here $c$ is a constant that allows to set the significance level of the test.

The Neyman-Pearson lemma (Huber and Strassen, 1973) states that this likelihood-ratio test is the most powerful test for a given significance level. A variant uses the log-likelihood ratio $\log \left(\Lambda\left(s, \widehat{X}^{1}(k), \widehat{X}^{2}(k)\right)\right.$. The Algorithm[2 uses (4) to compute the likelihood ratio. 


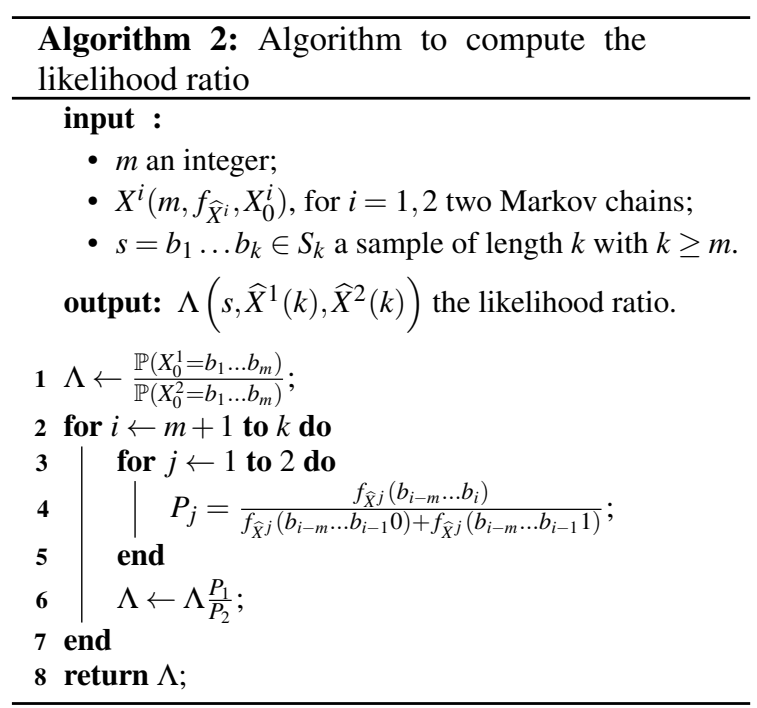

\subsection{Measuring the Relevance of the Test}

For $k \geq m$ an integer, the expected value of $\log \left(\Lambda\left(s, \widehat{X}^{1}(k), \widehat{X}^{2}(k)\right)\right.$, with $s$ drawn from $\widehat{X}^{1}(k)$ is (7). It is nothing but the Kullback-Leibler divergence (Kullback and Leibler, 1951, Hershey and Olsen, 2007) of $\widehat{X}^{1}(k)$ and $\widehat{X}^{2}(k)$, denoted by: $K L\left(\left(\widehat{X}^{1}(k) \| \widehat{X}^{2}(k)\right)\right.$. Thus, the Kullback-Leibler divergence gives an assessment of the distance between the distributions of $\widehat{X}^{1}(k)$ and $\widehat{X}^{2}(k)$. One has to remark that it is particularly well suited for the likelihood ratio test. The bigger the Kullback-Leibler divergence is, the more the test will be able to successfully distinguish $\widehat{X}^{1}(k)$ from $\widehat{X}^{2}(k)$.

When a statistical model for a corpus of very structure files is computed, it is not so uncommon that some states of a Markov chain are almost never or never reached. In the case where $\mathbb{P}\left(\widehat{X}^{2}(k)=s\right)=0$, $K L\left(\left(\widehat{X}^{1}(k) \| \widehat{X}^{2}(k)\right)\right.$ is not defined and the computation of the likelihood ratio may fail. Indeed, in the computation of likelihood ratio or in the KullbackLeibler divergence, there is the following quotient: for $k \geq m$ and $s \in S_{k}$ :

$$
\Lambda\left(s, \widehat{X}^{1}(k), \widehat{X}^{2}(k)\right)=\frac{\mathbb{P}\left(\widehat{X}^{1}(k)=s\right)}{\mathbb{P}\left(\widehat{X}^{2}(k)=s\right)} .
$$

Of course if $X^{2}\left(m, f_{\widehat{X}} 2, X_{0}^{2}\right)$ is the model corresponding to the random distribution, then, $\Lambda\left(s, \widehat{X}^{1}(s), \widehat{X}^{2}(s)\right)$ can always be computed. In this case, we can also compute the Kullback-Leibler divergence. But we would like to be able to compute the divergence between two not random distributions.
Furthermore, it may also happen that $\mathbb{P}\left(\widehat{X}^{2}(k)=\right.$ $s)$ is not zero but is very small, which corresponds to very rare states. In this case, the statistics computed during the training phase may happen to be not significant, since they have been computed with a very small sample. It could generate false negative for our test. One of the main point of the present paper is to explain how to deal with this situation by introducing a tweaked Kullback-Leibler divergence and use it to choose the best parameters for our Markov chain model.

\section{First Experiments and Results}

In this section, we make a first analysis of our corpus of files.

The given corpus is split into two parts, one is for training while the other is for testing the model. The purpose of this corpus is to make a model of legit and well-structured data that are commonly present on anybody's computer, as well as observing how already-known ransomware encrypt data.

The training corpus is composed of 10134 files with 49 different extensions. The extensions frequencies are shown in Figure 1 .

The model is built on file headers analyse. It is possible to group extensions by header format. For example: doc, docx, ppt, pptx, xls, xlsx share the same header format in Microsoft Office suite.

The test corpus is composed of 27 plaintexts files and their associated ciphertexts for different ransomware. The list of ransomware and their specific behaviour is shown on Table 1 .

\begin{tabular}{|l|l|}
\hline Ransomware & Behaviour \\
name & Encrypt and insert its own specific header just before the ciphertext \\
\hline Zppelin & Not specified \\
GlobImposter & Encrypt the whole file, only encrypt some files \\
Gigsaw & Encrypt headers \\
Paradise & Encrypt a part of the file (including the header) \\
Deadmin & Encrypt the whole file, do not encrypt .exe \\
Estemani & Encrypt the whole file by block of 8192 bytes \\
GlobImposter_half & Encrypt the whole file, do not encrypt .exe \\
MedusaLocker & Encrypt approximately the first 131072 bytes, do not encrypt .exe \\
NemtyRevenge & Encrypt and insert a padding except for . jpg, do not encrypt .exe \\
Ordinypt & Encrypt approximatively the first 151552 bytes \\
StopDjvuMoka & Encrypt approximatively the first 16384 bytes \\
Unknown_6rndchar & Encrypt the whole file, do not encrypt .exe \\
MegaCortex & Encrypt some files entirely, insert paddings of zeros in some others \\
Phobos & Encrypt $3 / 4$ of the file, move some sections, do not encrypt .exe \\
Sodinokibi & Encrypt the first 1048576 bytes \\
Eris & Encrypt the whole file \\
Maoloa & Encrypt the first 71680 bytes, do not encrypt .exe \\
Nemsis & Encrypt the whole file, does not encrypt .exe and .zip \\
Seon2 & Encrypt the whole file, but mostly conserve their original entropy \\
Xorist &
\end{tabular}

Table 1: List of of ransomware and their specific behaviour.

For the first experiments, we have trained multiple models on different file formats: jpg, gif, msoffice and pdf. These formats are the most present ones in 


$$
\mathbb{E}\left(\log \left(\Lambda\left(s, \widehat{X}^{1}(k), \widehat{X}^{2}(k)\right)\right)\right)=\sum_{s \in S_{k}} \mathbb{P}\left(\widehat{X}^{1}(k)=s\right) \cdot \log \left(\frac{\mathbb{P}\left(\widehat{X}^{1}(k)=s\right.}{\mathbb{P}\left(\widehat{X}^{2}(k)=s\right.}\right)
$$

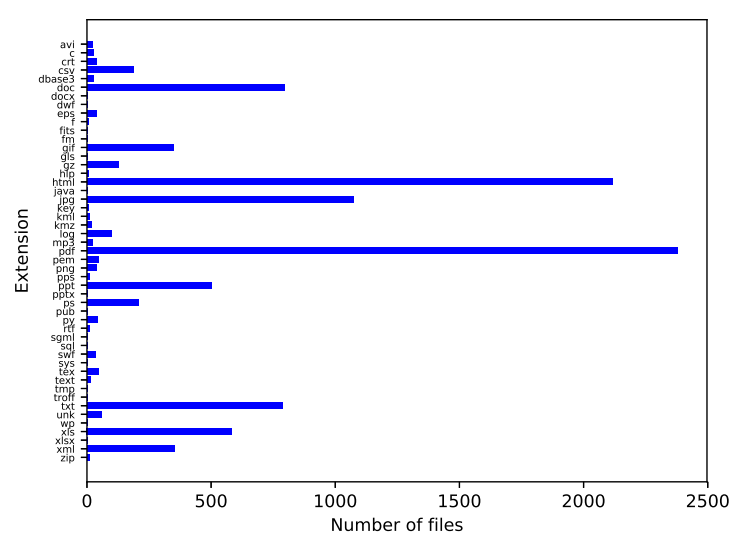

Figure 1: Number of files by extension in the training corpus.

the corpus. Indeed, we have considered subsets of the training corpus consisting of files with the same extensions because it seems reasonable to suppose that their file headers have the same structure that will be captured by the Markov chain model. We wanted to see how much our approach is sensitive to the type of files of the corpus.

The parameters for the experiments have been chosen based on the KL-divergence to randomness. We trained multiple models with different parameters $m$ (memory) and $N$ (header length). The parameter $N$ is ranging from 128 to 2048 , which seem to be reasonable sizes for file headers. The parameter $m$ is ranging from 4 to 16, a larger value would not be reasonable knowing the number of states in a Markov chain grows exponentially as the memory grows. The results are shown in Table 2

While the header length $N$ seems to have a significant effect on the divergence for each format, to increase in memory $m$ seems to globally decrease the divergence. Based on those observations and the results in Table 2, we have chosen the following parameters for our experiments: $m=4$ and $N=512$. Indeed, the choice for $m$ is obvious, we have decided to set $N=512$, since it maximizes the divergence for msoffice files.

To ensure the significance of the test, we have recorded the evolution of the KL-divergence while training the Markov chain model. The results are shown in Figure. 2. It is important to take a look at the evolution to ensure that the metric tends to converge to a non-null value. A null value would indicate that the test would be unable to distinguish the trained model from randomness. We can see in Figure 2 that the divergence seems to more or less converge in most cases.

The divergence of msoffice files is really high compared to other format. This is not surprising, this format is known to have a header that leaves small room for randomness. As for jpg and gif files, the divergence is relatively smaller and this is again not surprising because these formats do not have headers as static as msoffice. Finally, the divergence for $p d f$ files seems to be almost zero because the pdf headers are very varying.

The model trained over the whole corpus, without taking into account file extension, seems to converge to a divergence of approximately 0.069 .

\section{$5.1 \quad$ Test and Results}

To test the trained models against randomness, we have created for each format a corpus half composed of randomly generated files and half composed of files that match the format and were not in the original training corpus. We have used the likelihood ratio test (see Definition 4.1) with the constant $c=1$. The test is positive if it says that the sample corresponds to a structured header and negative if it says that the sample is random. The results are shown in Table 3 . One has to remark that the success rate of positive testcases for gif and pdf is not maximal. By looking at the computation of the likelihood ratio, we figured out the issue is due to rare states and transitions that are not highly present in the original corpus.

\subsection{Explanation of False Negatives}

The results showed that some positive test-cases are categorized as negative by the test. We figured out this behaviour is due to some states that are relatively little present in the original corpus.

Let $X\left(m, f_{\widehat{X}}, X_{0}\right)$ be a trained model, we say that $b_{1} \ldots b_{m} \in S_{m}$ is a rare state if (with the notations of Section 4.1): $c_{H_{N}}\left(b_{1} \ldots b_{m}\right)=c_{H_{N}}\left(b_{1} \ldots b_{m} 0\right)+$ $c_{H_{N}}\left(b_{1} \ldots b_{m} 1\right)$ is small compared to the total number of transitions in the corpus. Said in another way, a $s \in S_{m}$ is rare if

$$
f_{\widehat{X}}(s 0)+f_{\widehat{X}}(s 1)<t
$$

for a $t>0$ small. If $s \in S_{m}$ is a rare state it may happen 


\begin{tabular}{|c|c|c|c|c|c|c|c|c|c|c|}
\hline${ }_{m}{ }^{N}$ & \multicolumn{2}{|c|}{128} & \multicolumn{2}{|c|}{256} & \multicolumn{2}{|c|}{512} & \multicolumn{2}{|c|}{1024} & \multicolumn{2}{|c|}{2048} \\
\hline \multirow{4}{*}{4} & msoffice & 0.25 & msoffice & 0.30 & msoffice & 0.39 & msoffice & 0.25 & msoffice & 0.14 \\
\hline & jpeg & 0.13 & jpeg & 0.12 & jpeg & 0.10 & jpeg & 0.09 & jpeg & 0.11 \\
\hline & gif & 0.11 & gif & 0.12 & gif & 0.09 & gif & 0.06 & gif & 0.04 \\
\hline & pdf & 0.01 & pdf & 0.01 & pdf & 0.02 & pdf & 0.02 & pdf & 0.03 \\
\hline \multirow{4}{*}{8} & msoffice & 0.32 & msoffice & 0.32 & msoffice & 0.38 & msoffice & 0.23 & msoffice & 0.13 \\
\hline & jpeg & 0.11 & jpeg & 0.08 & jpeg & 0.08 & jpeg & 0.09 & jpeg & 0.11 \\
\hline & gif & 0.09 & gif & 0.10 & gif & 0.08 & gif & 0.06 & gif & 0.04 \\
\hline & pdf & 0.01 & pdf & 0.02 & pdf & 0.02 & pdf & 0.02 & pdf & 0.03 \\
\hline \multirow{4}{*}{16} & msoffice & 0.29 & msoffice & 0.32 & msoffice & 0.33 & msoffice & 0.17 & msoffice & 0.09 \\
\hline & jpeg & 0.05 & jpeg & 0.03 & jpeg & 0.03 & jpeg & 0.04 & jpeg & 0.06 \\
\hline & gif & 0.06 & gif & 0.06 & gif & 0.05 & gif & 0.04 & gif & 0.02 \\
\hline & pdf & 0.00005 & pdf & 0.0003 & pdf & 0.0004 & pdf & 0.0004 & pdf & 0.0002 \\
\hline
\end{tabular}

Table 2: KL-divergence for different choices of parameters $m$ (memory) and $N$ (header length), and for different file formats

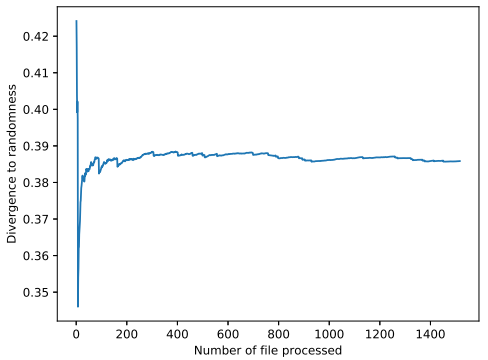

(a) Evolution of the divergence for msoffice files

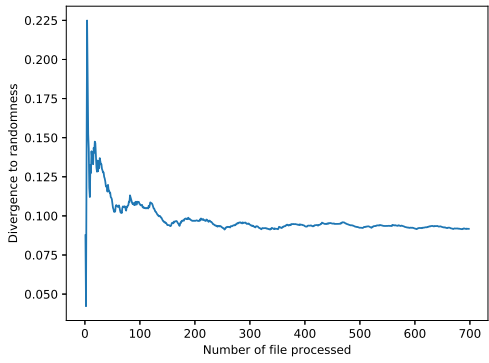

(c) Evolution of the divergence for gif files

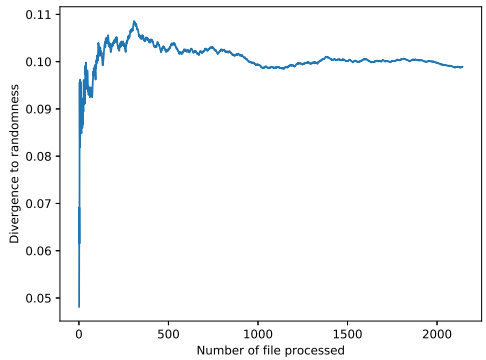

(b) Evolution of the divergence for ineg files

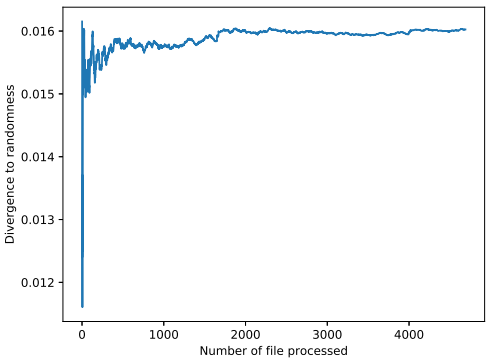

(d) Evolution of the divergence for pdf files Figure 2: Evolution of the divergence for different file formats $(m=4, N=512)$

\begin{tabular}{|l|l|l|l|l|l|}
\hline Format & \# true positive & \# true negative & \# false negative & \# false positive & Accuracy \\
\hline msoffice & 330 & 330 & 0 & 0 & 1.00 \\
\hline jpeg & 330 & 330 & 0 & 0 & 1.00 \\
\hline pdf & 322 & 330 & 8 & 0 & 0.98 \\
\hline gif & 142 & 150 & 8 & 0 & 0.97 \\
\hline
\end{tabular}

Table 3: First results of tests against randomness for multiple file formats

that

$$
\left\{\begin{array}{l}
c_{H_{N}}\left(b_{1} \ldots b_{m} 0\right)=0 \\
\text { or } \\
c_{H_{N}}\left(b_{1} \ldots b_{m} 1\right)=0
\end{array}\right.
$$

As a result, when such a state is encountered in a testcase and the transition taken is the one of null probability, the numerator of the likelihood ratio becomes zero and eventually the file is categorised as negative. Since the global number of states is $2^{m}$, we observed this behaviour to be more significant on the results with greater values of $m$. For instance the proportion of false negative was about $33 \%$ for $m=16$.

The issue is that the test only takes into account the transition probabilities, it does not take into account whether the state's count in the corpus is representative or not compared to other's state counts. For example, it is irrelevant to reject a file only because of one state whose count in the corpus is only 4 while the total number of transitions seen in the corpus is greater than 400000 . This means that in this case the decision is taken on little information which only rep- 
resent $\frac{1}{100000}$ of the original corpus, this is like deducing generalities from specific cases.

\section{Refine the Models and Tests}

In this section we describe the solutions implemented to overcome the issue of rare states. A modification of the model is introduced to involve applying a threshold to get rid of irrelevant information.

\subsection{Adding a Threshold}

The main idea is to get rid of irrelevant information, by applying a threshold on state probabilities in the trained model $X\left(m, f_{\widehat{X}}, X_{0}\right)$. That is to say, for a threshold $t \in[0,1], \forall b_{1} \ldots b_{m} \in\{0,1\}^{m}$, during the learning phase, we set:

$f_{\widehat{X}}\left(b_{1} \ldots b_{m+1}\right)= \begin{cases}\frac{1}{2} \frac{c_{H_{N}}\left(b_{1} \ldots b_{m}\right)}{\left|H_{N}\right|(m)} & \text { if } \frac{c_{H_{N}}\left(b_{1} \ldots b_{m}\right)}{\left|H_{N}\right|(m)}<t \\ \frac{c_{H_{N}}\left(b_{1} \ldots b_{m+1}\right)}{\left|H_{N}\right|(m+1)} & \text { otherwise }\end{cases}$

Intuitively, we remove any information based on rare states, with $t$ a tolerance coefficient. We deduce Algorithm 3 to compute a Markov chain model of a data stream taking into account $t$.

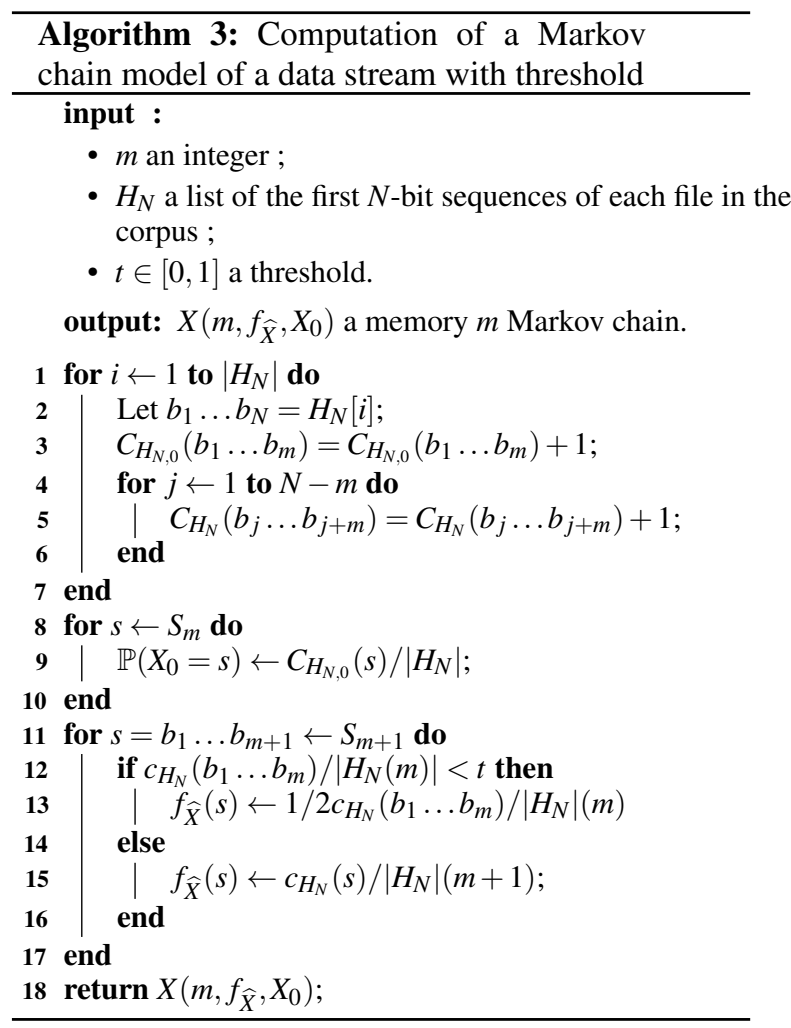

\subsection{Threshold Benefits}

In other words, once the likelihood ratio is computed, we can distinguish between the transitions which are relevant for the test from those who are not.

Formally, one can define some kind of confidence ratios based on the number of relevant transitions the likelihood ratio is computed with in line 6 of Algorithm 2. For this, let $s \in S_{N}$ be a header sample, we want to test this sample for a model $X^{1}\left(m, f_{\widehat{X}^{1}}, X_{0}^{1}\right)$ with threshold $t$ against a model $X^{2}\left(m, f_{\widehat{X}^{2}}, X_{0}^{2}\right)$ with the same threshold $t$.

We write $s=b_{1} \ldots b_{N}$ and we introduce:

$$
\Gamma_{X^{1}, X^{2}, t}(s)=\left(\frac{\gamma_{X^{1}, t}(s)}{N-m}, \frac{\gamma_{X^{2}, t}(s)}{N-m}\right),
$$

with $(8)$. It is clear that $\Gamma_{X^{1}, X^{2}, t}(s)$ is the pair of proportions of relevant transitions in the sample $s$ for both models $X^{1}$ and $X^{2}$. The higher these values are, the more relevant the test should be. The Algorithm 4 computes the likelihood ratio of a sample $s \in S_{k}$ and outputs $\Gamma_{X^{1}, X^{2}, t}(s)$ taking into account a certain threshold $t$.

We remark that if we increase $t$ the proportion of relevant transition in the computation of a sample $s$ will decrease but if a sample has only relevant transitions with respect to $t$, we will be confident that the likelihood ratio is meaningful from a statistical point of view. In order to choose $t$, we can compute the expectancy, that we denote by $\mathbb{E}(\widehat{X}, t)$, that a sample $s \in S_{N}$ drawn following the distribution $\widehat{X}(N)$ has only relevant transitions that is:

$$
\mathbb{E}(\widehat{X}, t)=\sum_{s=b_{1} \ldots b_{N} \in S_{N}} \mathbb{P}(\widehat{X}(N)=s) T_{\widehat{X}(m)}(s),
$$

where $T_{\widehat{X}(N)}: S_{N} \rightarrow\{0,1\}$ is the function such that

- $T_{\widehat{X}(N)}(s)=0$ for $s=b_{1} \ldots b_{N}$ if there is a $i \in$ $\{1, \ldots, N-m+1\}$ such that $f_{\widehat{X}}\left(b_{i} \ldots b_{i+m-1} 0\right)+$ $f_{\widehat{X}}\left(b_{i} \ldots b_{i+m-1} 1\right)<t$

- and $T_{\widehat{X}(N)}(s)=1$ otherwise.

Then one can choose a $\mathbb{E}(\widehat{X}, t)$ which give the probability that a test will be relevant which fixes a $t$.

\subsection{Results}

The same tests as in Section 5.1, have been ran, on the same corpus, with threshold models for pdf and gif formats. The results for the best observed $t$ parameter are shown in Table 4

The results are optimal for pdf files, the threshold achieves a success rate of $100 \%$. However, it 


\begin{tabular}{|l|l|l|l|l|l|l|}
\hline Format & $t$ & \# true positive & \# true negative & \# false negative & \# false positive & Accuracy \\
\hline pdf & 0.05 & 330 & 330 & 0 & 0 & 1.00 \\
\hline gif & 0.05 & 142 & 150 & 8 & 0 & 0.97 \\
\hline
\end{tabular}

Table 4: Results of tests with thresholded models against randomness for pdf and gif files $(m=4, N=512)$

\begin{tabular}{|l|l|l|l|l|l|l|}
\hline Format & $t$ & \# true positive & \# true negative & \# false negative & \# false positive & Accuracy \\
\hline pdf & 0.0005 & 330 & 330 & 0 & 0 & 1.00 \\
\hline gif & 0.05 & 149 & 150 & 1 & 0 & 0.99 \\
\hline
\end{tabular}

Table 5: Results of tests with thresholded models against randomness for pdf and gif files $(m=16, N=512)$

$$
\gamma_{X, t}=\left|\left\{s=b_{i} \ldots b_{i+m-1} \in S_{m}, i=1, \ldots, N-m+1 \mid f_{\widehat{X}}(s 0)+f_{\widehat{X}}(s 1) \geq t\right\}\right| .
$$

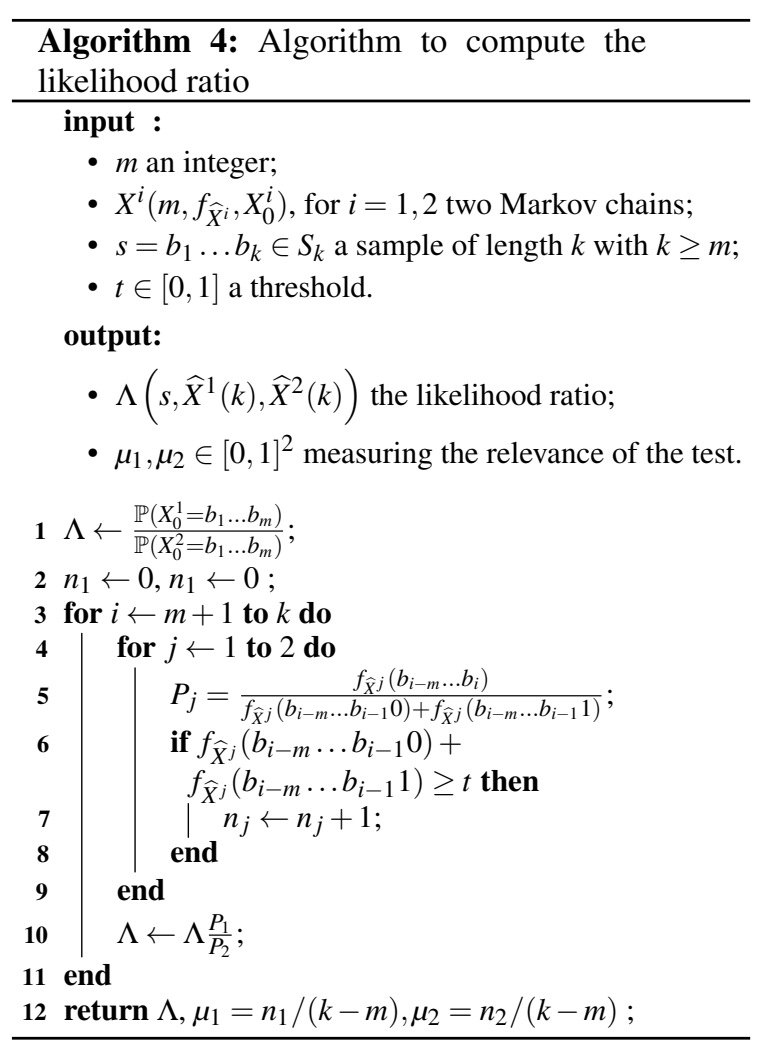

does not work so well for gif files, the threshold does not improve the success rate. This is not surprising because with $m=4$, the observed transitions are only distributed over a set of $2^{4}=16$ states. This does not allow for a lot of precision in the choice of the threshold since we are basically ignoring a whole state, when $m=4$ this represents $\frac{1}{16}$ of the et of states.

According to the previous observation, it should be easier to find a good threshold for greater $m$. Thus, the threshold model has also been tested with different values of $m$. The results are the best for $m=16$ as shown in Table 5 .

\section{Experiments on Real-World Ransomware}

In this section we show and analyse the results of our tests against files encrypted by real-world ransomware. We used a model trained over the whole corpus for these tests. It is important to precise that the ransomware are active only a short moment, so it is difficult to compare two experimentations paper.

\subsection{Models and Parameters}

As done in section 5, we compared the divergence to randomness for different values of $m$ and $N$ and selected the ones that maximize the metric. The results are shown in Table 6. According to the diver-

\begin{tabular}{|c|c|c|c|c|c|}
\hline$m$ & 128 & 256 & 512 & 1024 & 2048 \\
\hline 4 & 0.044 & 0.057 & 0.069 & 0.049 & 0.035 \\
\hline 8 & 0.074 & 0.074 & 0.084 & 0.060 & 0.045 \\
\hline 16 & 0.055 & 0.062 & 0.067 & 0.040 & 0.025 \\
\hline
\end{tabular}

Table 6: Divergence to randomness (whole corpus) for different parameters $m$ and $N$

gence results we have selected the following parameters: $m=8$ and $N=512$.

\subsection{Results and Analysis}

We have run a test for each ransomware in the corpus, on both encrypted files (we removed the files that were not encrypted by the ransomware) and original files. The results are shown in Table 7 . The tested ransomware all give two false negatives. The formats of those two files .exe and $.7 z$ are actually not present in the corpus, thus we do not expect them to be tested positive. That means the accuracy in most cases is probably the maximum we can expect from the model.

However, there are still significant issues with some specific ransomware. Orinypt \& Phobos: the 


\begin{tabular}{|l|l|l|l|l|l|}
\hline Ransomware & \# true positive & \# true negative & \# false negative & \# false positive & Accuracy \\
\hline Gigsaw & 25 & 16 & 2 & 0 & 0.95 \\
\hline Ordinypt & 25 & 4 & 2 & 20 & 0.57 \\
\hline Maoloa & 25 & 27 & 2 & 0 & 0.96 \\
\hline MedusaLocker & 25 & 24 & 2 & 0 & 0.96 \\
\hline Phobos & 25 & 12 & 2 & 15 & 0.69 \\
\hline Unknown & 25 & 27 & 2 & 0 & 0.96 \\
\hline Nemsis & 25 & 24 & 2 & 0 & 0.96 \\
\hline Deadmin & 25 & 27 & 2 & 0 & 0.96 \\
\hline Seon2 & 25 & 23 & 2 & 0 & 0.96 \\
\hline Zppelin & 25 & 27 & 2 & 0 & 0.96 \\
\hline Paradise & 25 & 27 & 2 & 0 & 0.96 \\
\hline StopDjvuMoka & 25 & 27 & 2 & 0 & 0.96 \\
\hline MegaCortex & 25 & 24 & 2 & 0 & 0.96 \\
\hline NemtyRevenge & 25 & 23 & 2 & 0 & 0.96 \\
\hline Globlmposter & 25 & 22 & 2 & 5 & 0.87 \\
\hline Globlmposter_half & 25 & 27 & 2 & 0 & 0.96 \\
\hline Estemani & 25 & 24 & 2 & 0 & 0.96 \\
\hline Sodinokibi & 25 & 24 & 2 & 0 & 0.96 \\
\hline Eris & 25 & 27 & 2 & 0 & 0.96 \\
\hline xorist & 25 & 2 & 2 & 25 & 0.5 \\
\hline
\end{tabular}

Table 7: Results with a model trained over the whole corpus tested against ransomware encrypted files $(m=8, N=512)$

issues with these two ransomware is that they put a padding of zeroes at the beginning of some encrypted files, since the transition from the all-zero state to itself is quite common in the corpus and can also be an initial state, the model has troubles distinguishing it. xorist: we believe the issues with this one is due to its ability to conserve more or less the original entropy of encrypted files, thus it does not fit into our model of encrypted data based on randomness.

As we can see, the main issue is that some ransomware encryption techniques do not fit into our model of encrypted data. A possible way to solve these issues would be to train a model over a large number of files encrypted with those ransomware and then perform a test against these newly trained models. However, this would ruin the preventive aspect of this approach since these specific ransomware would have to be known before being detectable.

\section{Conclusion and Future Works}

We have introduced a new countermeasure based on Markov Chains to detect ransomware based on their encryption behaviour. We also define a metric associated to our models to give an idea on how well the test should work. Considering ransomware use semantically secure encryption schemes, we represent encrypted data by a complete randomn Markov chain model and are able to distinguish the structured files from random data. According to our observations we have refined our approach and models to fix potential issues and get better results. Finally, we have proved our approach to be applicable to real-world cases by successfully distinguishing structured files from files encrypted with known ransomware whose encryption schemes fit into our models.

While the results are quite encouraging, there are still some points to be dealt with more in depth. We have mentioned that all the metrics do not take into account the initial state probabilities, defining new metrics to fix this issue would help getting a more precise idea on the significance of the tests. Finally, all tests on ransomware were made on small corpora, running a full benchmark on thousands of files would be needed to ensure the reliability of the approach.

One limitation of this work is that it cannot detect a ransomware which do not encrypt header of files. This countermeasure is not enough on her own. But it's a pertinent additional tool to complete other implemented countermeasures against ransomware. In the fight against ransomware, more indicators we have, better is the detection. Moreover, the Markov chain models have a low cost in terms of computing. So they can be deployed in the kernel to stop malicious processes without slowing down too much the computer.

Acknowledgments The authors would like to thank Jean-Louis Lanet and Aurélien Palisse for their for their helpful comments and discussions.

\section{REFERENCES}

Ahmadian, M. M., Shahriari, H. R., and Ghaffarian, S. M. (2015). Connection-monitor \& connection-breaker: A novel approach for prevention and detection of high survivable ransomwares. In (ISCISC).

Akcora, C. G., Li, Y., Gel, Y. R., and Kantarcioglu, M. (2019). Bitcoinheist: Topological data analysis for ransomware detection on the bitcoin blockchain. arXiv preprint.

Al-rimy, B. A. S., Maarof, M. A., Prasetyo, Y. A., Shaid, S. Z. M., and Ariffin, A. F. M. (2018a). Zero-day aware decision fusion-based model for crypto-ransomware early detection. International Journal of Integrated Engineering.

Al-rimy, B. A. S., Maarof, M. A., and Shaid, S. Z. M. (2018b). Ransomware threat success factors, taxonomy, and countermeasures: A survey and research directions. Computers \& Security.

Al-rimy, B. A. S., Maarof, M. A., and Shaid, S. Z. M. (2019). Crypto-ransomware early detection model using novel incremental bagging with enhanced semirandom subspace selection. Future Generation Computer Systems.

Almashhadani, A. O., Kaiiali, M., Sezer, S., and O'Kane, P. (2019). A multi-classifier network-based crypto ransomware detection system: a case study of locky ransomware.

Ash, R. B. (1990). Information theory. Dover Publications, Inc., New York. Corrected reprint of the 1965 original.

Aurangzeb, S., Aleem, M., Iqbal, M. A., Islam, M. A., et al. (2017). Ransomware: a survey and trends. J. Inf. Assur. Secur. 
Baykara, M. and Sekin, B. (2018). A novel approach to ransomware: Designing a safe zone system. In ISDFS. IEEE.

Cabaj, K., Gregorczyk, M., and Mazurczyk, W. (2018). Software-defined networking-based crypto ransomware detection using http traffic characteristics. Computers \& Electrical Engineering.

Castiglione, J. and Pavlovic, D. (2019). Dynamic distributed secure storage against ransomware. IEEE Transactions on Computational Social Systems.

Chen, Z.-G., Kang, H.-S., Yin, S.-N., and Kim, S.-R. (2017). Automatic ransomware detection and analysis based on dynamic api calls flow graph. In Proceedings of the International Conference on Research in Adaptive and Convergent Systems.

Continella, A., Guagnelli, A., Zingaro, G., Pasquale, G. D., Barenghi, A., Zanero, S., and Maggi, F. (2016). ShieldFS: A Self-healing, Ransomware-aware Filesystem. In ACSAC. ACM.

Genç, Z. A., Lenzini, G., and Ryan, P. Y. (2018). Next generation cryptographic ransomware. In Nordic Conference on Secure IT Systems. Springer.

Hershey, J. R. and Olsen, P. A. (2007). Approximating the kullback leibler divergence between gaussian mixture models. In IEEE-ICASSP.

Huber, P. J. and Strassen, V. (1973). Minimax tests and the neyman-pearson lemma for capacities. The Annals of Statistics.

Katz, J. and Lindell, Y. (2007). Introduction to Modern Cryptography (Chapman \& Hall/Crc Cryptography and Network Security Series). Chapman \& Hall/CRC.

Kharraz, A., Arshad, S., Mulliner, C., Robertson, W. K., and Kirda, E. (2016). UNVEIL: A Large-Scale, Automated Approach to Detecting Ransomware. In USENIX.

Kharraz, A. and Kirda, E. Redemption: Real-Time Protection Against Ransomware at End-Hosts. In RAID 2017.

Kullback, S. and Leibler, R. A. (1951). On information and sufficiency. Ann. Math. Statistics.

Lee, K., Lee, S.-Y., and Yim, K. (2019). Machine learning based file entropy analysis for ransomware detection in backup systems.

Moore, C. (2016). Detecting ransomware with honeypot techniques. In 2016 Cybersecurity and Cyberforensics Conference (CCC). IEEE.

Moussaileb, R. (2020). Log Analysis for Malicious Software Detection. $\mathrm{PhD}$ thesis.

Moussaileb, R., Bouget, B., Palisse, A., Le Bouder, H., Cuppens, N., and Lanet, J.-L. (2018). Ransomware's early mitigation mechanisms. In Proceedings of the 13th International Conference on Availability, Reliability and Security. ACM.

Moussaileb, R., Cuppens, N., Lanet, J.-L., and Le Bouder, H. (2019). Ransomware network traffic analysis for pre-encryption alert. In FPS2019.

Palisse, A., Durand, A., Le Bouder, H., Le Guernic, C., and Lanet, J.-L. (2017). Data aware defense (dad): towards a generic and practical ransomware countermeasure. In Nordic Conference on Secure IT Systems. Springer.

Palisse, A., Le Bouder, H., Lanet, J.-L., Le Guernic, C., and Legay, A. (2016). Ransomware and the legacy crypto api. In International Conference on Risks and Security of Internet and Systems. Springer.

Patton, M. W., Scott, N., Gutierrez, R. R., and Giovannini, S. (2019). Behavior-based ransomware detection using decoy files.

Pont, J., Arief, B., and Hernandez-Castro, J. (2020). Why current statistical approaches to ransomware detection fail. In International Conference on Information Security. Springer.

Scaife, N., Carter, H., Traynor, P., and Butler, K. R. B. (2016). CryptoLock (and Drop It): Stopping Ransomware Attacks on User Data. In ICDCS. IEEE.

Yassine Lemmou, Hélène Le Bouder, J.-L. L. (2019). Discriminating unknown software using distance model. 